\title{
Revestimiento interno en reconstrucción nasal: Estudio anatómico cadavérico
}

\author{
Internal lining in nasal reconstruction: A cadaveric anatomical study
}

Fernando Casanueva L ${ }^{1}$, José Alzérreca A $^{2}$.

\begin{abstract}
RESUMEN
Introducción: Ante la presencia de un defecto anatómico nasal, se hace necesario considerar alternativas para restituir una correcta función y estética nasal. La estructura osteocartilaginosa nasal debe contar con un soporte o revestimiento interno que aporte una vascularización necesaria. Existen diversas técnicas de colgajos intranasales para lograr reconstituir el revestimiento interno nasal.

Objetivos: Describir la técnica quirúrgica de los principales colgajos de revestimiento interno en reconstrucción nasal y su aplicación en modelos ex vivo.

Material y método: Se realizó la disección de 7 especímenes de donante cadáver. Se efectuó una resección amplia nasal simulando una pérdida de tejido de las 3 capas de la anatomía nasal para su posterior reconstrucción.

Resultados: Se logró replicar las distintas alternativas de técnicas de colgajos intranasales descritas para reconstrucción nasal.

Conclusión: El revestimiento interno es de suma importancia en la reconstrucción nasal. Esta es una primera fase en el desarrollo y aprendizaje de la reconstrucción nasal.

Palabras clave: Reconstrucción nasal, colgajos nasales, revestimiento interno.
\end{abstract}

\begin{abstract}
Introduction: In the presence of a nasal anatomical defect, it is necessary to consider alternatives to restore a correct function and esthetic nasal result. The nasal osteocartilaginous structure must have an internal support or lining that provides a necessary vascularization. There are various techniques of intranasal flaps to achieve reconstitution of the nasal internal lining.

Aim: To describe the surgical technique of the main internal lining flaps in nasal reconstruction and its application in ex vivo models.

Material and method: We dissected 7 cadaver donor specimens. A broad nasal resection was performed simulating a loss of tissue from the three layers of the nasal anatomy for subsequent reconstruction.
\end{abstract}

Médico del Servicio de Otorrinolaringología. Hospital San Vicente. Arauco

2 Médico del Servicio de Otorrinolaringología. Hospital Guillermo Grant Benavente. Concepción.

Recibido el 30 de enero, 2017. Aceptado el 21 de marzo, 2017. 
Results: It was possible to replicate the different alternatives of intranasal flap techniques described for nasal reconstruction.

Conclusion: The inner lining is of paramount importance in nasal reconstruction. This is a first phase in the development and learning of nasal reconstruction.

Key words: Nasal reconstruction, nasal flaps, internal lining.

\section{INTRODUCCIÓN}

Cuando existe una pérdida de la anatomía nasal, ésta debe ser reestructurada por completo, idealmente con tejido similar al deficiente, para lograr una adecuada recuperación funcional y estética ${ }^{1}$.

Las causas de una pérdida de la anatomía nasal son variadas, destacando el trauma, específicamente las mordidas de perro en niños, y las resecciones tumorales en adultos ${ }^{2,3}$. Actualmente la incidencia de cáncer de piel se encuentra en aumento exponencial debido a la exposición solar, por lo que la cirugía de reconstrucción nasal será cada vez más frecuente ${ }^{4}$.

Cuando pensamos en reconstrucción nasal, inmediatamente recordamos el colgajo frontal más conocido como colgajo indiano; éste ha sido y se mantiene como la técnica de elección desde incluso antes de Cristo ${ }^{5}$. Antiguamente las reconstrucciones nasales eran realizadas solo con cobertura de piel, pero posteriormente presentaban retracciones, colapso de la vía aérea y estenosis, debido a la falta de sostén anatómico de la nariz .

La anatomía normal nasal está compuesta de tres capas principales: piel, hueso/cartílago y mucosa. Al realizar una reconstrucción nasal se debe intentar reconstituir estas 3 capas ${ }^{3,6}$. Las dos capas externas de piel y la estructura osteocartilaginosa son reconstituidas con relativa mayor facilidad; lamentablemente reconstruir el revestimiento interno es una tarea mucho más compleja.

Los primeros intentos en reconstruir el revestimiento interno fueron realizados con injertos de piel o colgajos locales de piel, no obstante, éstos no tenían la vascularización necesaria llevando a necrosis del revestimiento interno, lo que generaba exposición, infección y extrusión del cartílago utilizado ${ }^{6}$. Por este motivo, la técnica a utilizar en la reconstrucción del revestimiento interno, debe presentar la misma característica de la capa interna en la anatomía nasal normal: Su profusa vascularización.

La relación entre el soporte y el revestimiento interno fue descrita como un "romance" por Burget $^{6}$. Esta llamativa descripción hace referencia a la dependencia que existe entre ellos; la viabilidad del injerto cartilaginoso depende de un revestimiento interno vascularizado, como el revestimiento interno necesita del soporte entregado por el injerto cartilaginos $0^{6}$.

En los ochenta hubo una revolución en el desarrollo de colgajos intranasales para reconstruir el revestimiento interno, estos colgajos se basan en arterias específicas capaces de entregar una vascularización necesaria, además de ser delgados y flexibles; favoreciendo de esta forma una reconstrucción más armoniosa ${ }^{6}$.

\section{OBJETIVOS}

El presente estudio tiene por objeto describir la técnica quirúrgica de los principales colgajos de revestimiento interno en reconstrucción nasal y su aplicación en modelos ex vivo.

\section{MATERIAL Y MÉTODO}

Se realizó un trabajo de disección de 7 especímenes de donante cadáver, éstas se realizaron en el centro de simulación y entrenamiento VirtuOHSU de la Oregon Health and Science University (OHSU). Las disecciones fueron realizadas por el autor principal. Se efectuó una resección amplia nasal simulando una pérdida de tejido de las 3 capas de la anatomía normal nasal, para su posterior reconstrucción. En los diferentes especímenes, se realizaron modelos de reconstrucción con las distintas posibilidades de reconstrucción de revestimiento interno. 


\section{RESULTADOS}

Como resultados de este trabajo se describen los diferentes colgajos realizados en los especímenes de donante cadáver.

\section{Colgajo de avance bipediculado de piel vestibular}

Aquellas lesiones vestibulares que comprometan el revestimiento interno menores de $1,5 \mathrm{~cm}$ pueden ser reparadas con este colgajo, ya sea uni o bilateral ${ }^{1}$. Primero se realiza una incisión similar al abordaje intercartilaginoso con una mayor extensión lateral. La piel vestibular es disecada y movilizada caudalmente (Figura 1). La zona donante es posteriormente reparada con injerto de piel. El colgajo debe ser suturado logrando una mínima tensión y sobre éste debe posicionarse el soporte estructural, ya sea cartílago septal 0 auricular ${ }^{1}$.

\section{Colgajo de mucopericondrio septal en bisagra ipsilateral}

Defectos alares superiores a $1,5 \mathrm{~cm}$ verticalmente no pueden ser reparados con el colgajo descrito anteriormente, ya que no hay suficiente piel entre la incisión intercartilaginosa y el defecto. Estas alteraciones pueden ser reparadas satisfactoriamente con un colgajo de mucopericondrio septal' ${ }^{1}$.
Éste debe ser diseñado lo más largo posible (4,5 $\mathrm{cm}$ vs $3 \mathrm{~cm}$ ), para permitir una reconstrucción satisfactoria del revestimiento interno afectado (Figura $2 \mathrm{~A})$. Este colgajo es de tipo circular por lo que su largo debe ser incluso mayor al doble del defecto

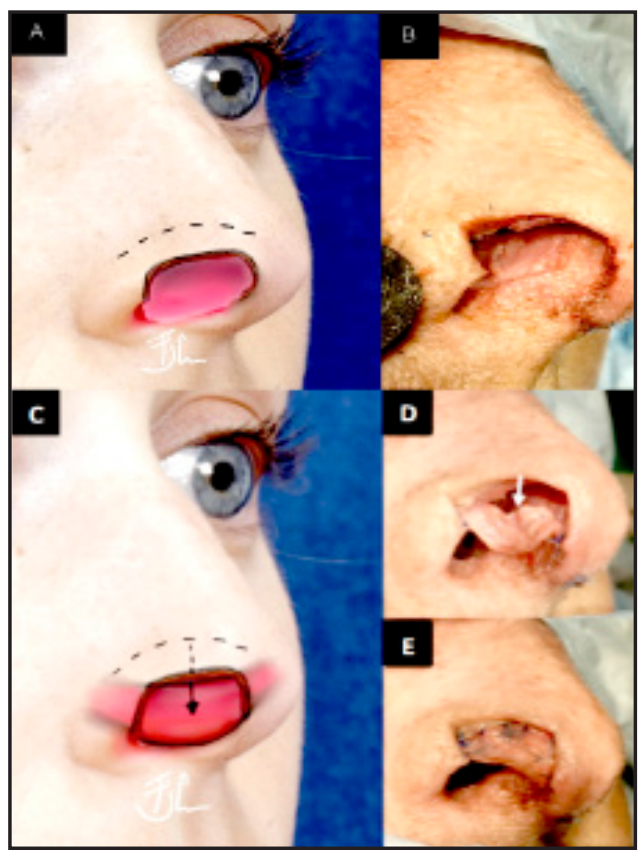

Figura 1. Colgajo de avance bipediculado de piel vestibular: 1A-1B: Incisión intercartilaginosa extendida lateralmente. 1C1D: Movilización caudal del colgajo reparando el revestimiento interno del defecto vestibular. 1E: Sutura del colgajo a remanente de revestimiento interno mucoso.

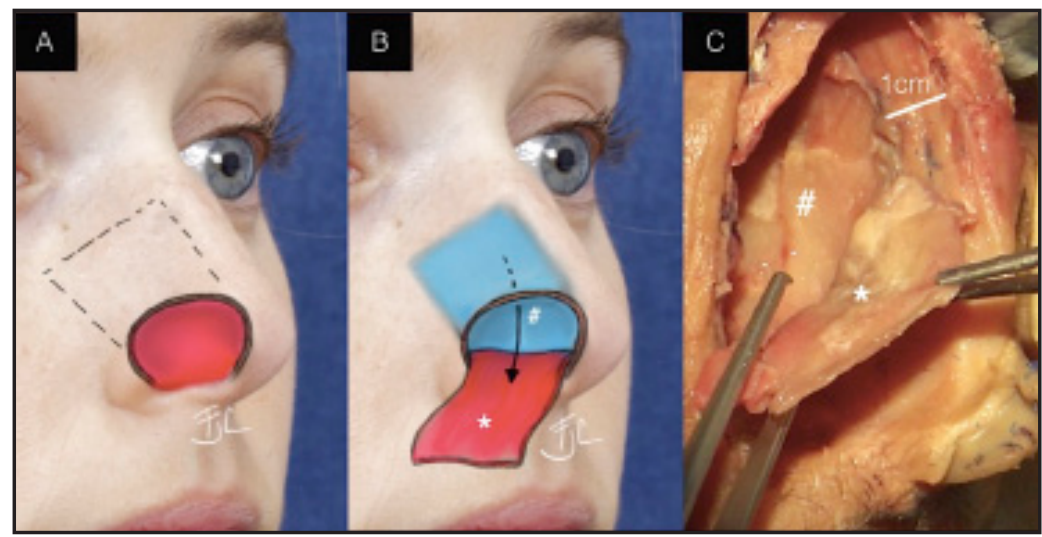

Figura 2. Colgajo de mucopericondrio septal en bisagra ipsilateral. 2A: El colgajo es diseñado con al menos $4 \mathrm{~cm}$ de longitud, la incisión longitudinal superior debe iniciarse a $0,75 \mathrm{~cm}$ aprox. del ángulo septal anterior y extenderse a 0,5-1 cm bajo el techo1. La incisión longitudinal inferior parte a $1 \mathrm{~cm}$ posterior a la espina extendiéndose por la unión de la cresta y piso de la fosa nasal. 2B-2C: El mucopericondrio ( $\left.{ }^{\star}\right)$ es cuidadosamente disecado del septum (\#). La incisión posterior necesita de un bisturí ángulo recto para su realización. La disección del mucopericondrio se extiende hasta aprox. 1,5 cm posterior a la espina y borde caudal del septum. 
a reparar'. El mucopericondrio es obtenido y cuidadosamente disecado del septum (Figura 2B) el cual se cubrirá de una capa de epitelio por segunda intención. Si es necesario reconstruir la estructura cartilaginosa, el septum cartilaginoso puede ser utilizado (Figura 3A) así como cartílago auricular, dependiendo del defecto.

El colgajo de mucopericondrio es movilizado lateralmente y suturado al defecto reconstituyendo de esta forma el revestimiento interno (Figura 3A, 3B).

\section{Colgajo de mucopericondrio septal en bisagra dorsal contralateral}

Colgajo compuesto por mucopericondrio septal rotado para la punta y columela. Este colgajo se obtiene del lado contralateral a la lesión y puede ser diseñado para incluir toda la mucosa que cubre el septum cartilaginoso, exceptuando el margen caudal y dorsal'. El cartílago septal expuesto es retirado posteriormente para injertos, manteniendo el soporte dorsal adecuado. Este colgajo es utilizado siempre en combinación con un colgajo de mucopericondrio septal en bisagra ipsilateral, por lo que el desplazamiento lateral hacia el defecto se realiza a través de la perforación septal resultante (Figura 4). El colgajo de mucosa se refleja lateralmente desde la línea media hacia el sitio del defecto y se sutura al colgajo de mucosa ipsilateral logrando un sostén vascular amplio para los injertos de cartílago posteriores (Figura 5).

\section{Colgajo compuesto condromucoso septal rotado}

Este colgajo está indicado en defectos grandes de la nariz ubicados en línea media, que incluyen lesiones en conjunto de punta y columela 0 del dorso nasal asociados 0 no a defectos de la pared lateral. Cuando la columela y la punta nasal están afectadas, este colgajo compuesto condromucoso septal provee la estructura cartilaginosa necesaria para la reconstrucción de la columela y el revestimiento interno del tabique. Este colgajo

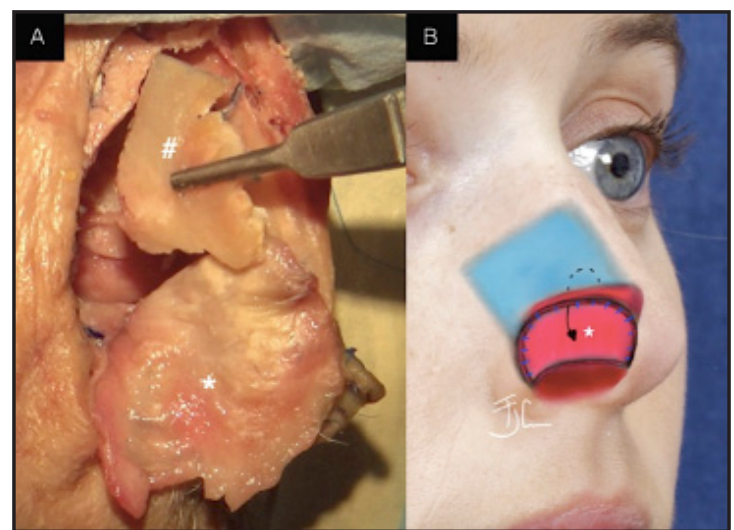

Figura 3. Colgajo de mucopericondrio septal en bisagra ipsilateral. 3A-3B: El colgajo de mucopericondrio $\left({ }^{*}\right)$ es movilizado lateralmente y suturado al defecto reconstituyendo de esta forma el revestimiento interno. Si es necesario el cartílago septal $\left(^{*}\right)$ puede ser obtenido con el fin de reconstruir la estructura cartilaginosa.

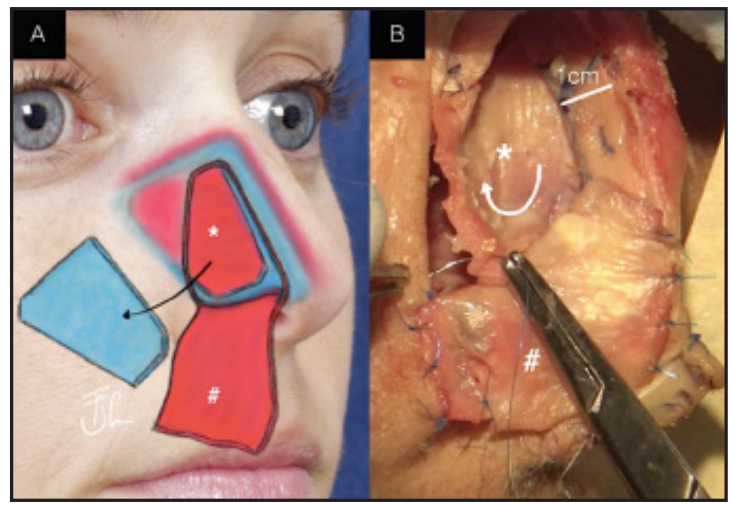

Figura 4. Colgajo de mucopericondrio septal en bisagra dorsal contralateral. 4A-4B: Este colgajo siempre va en combinación con uno de mucopericondrio septal en bisagra ipsilateral (\#). El mucopericondrio contralateral $\left(^{*}\right)$ se moviliza a través de la perforación septal generada al retirar el cartílago para injertos cartilaginosos asociados.

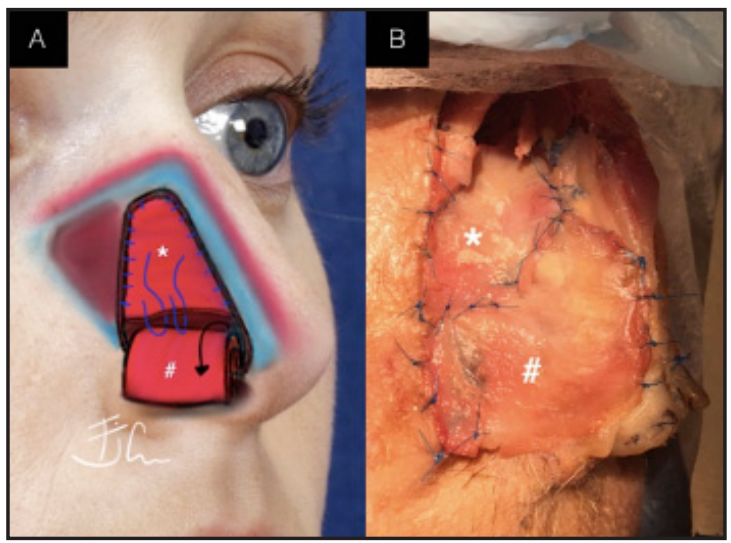

Figura 5. Colgajo de mucopericondrio septal en bisagra dorsal contralateral. 5A-5B: Sutura de colgajo de mucopericondrio ipsilateral (\#) y mucopericondrio contralateral $\left({ }^{*}\right)$ trasladado a través de línea media, logrando una estructura vascular de sostén para injertos de cartílagos posteriores. 


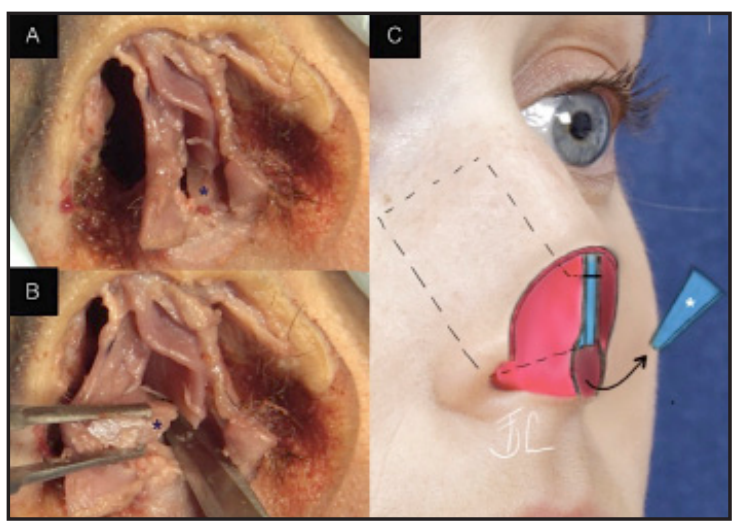

Figura 6. Colgajo compuesto condromucoso septal rotatorio. 6A-B-C: Utilizando un osteótomo se reseca un segmento de tabique $\left({ }^{\star}\right)$ para facilitar la posterior rotación del colgajo.

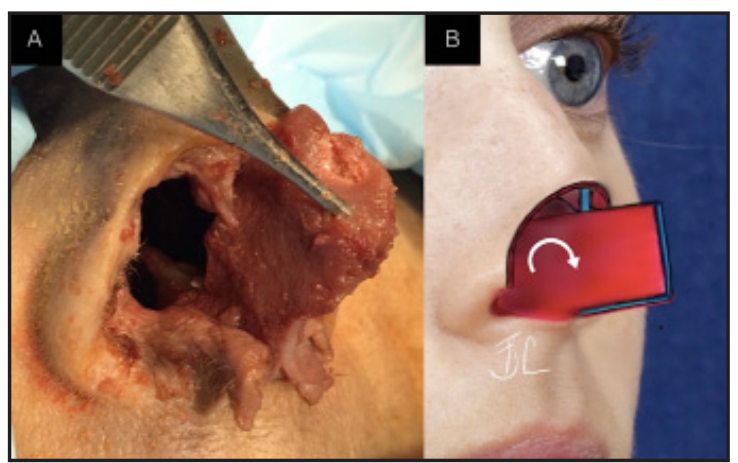

Figura 7. Colgajo compuesto condromucoso septal rotatorio. 7A-B: El colgajo es rotado en $90^{\circ}$ desde su base en dirección antero caudal hasta que el borde inferior del colgajo se ubica contra el remanente del marco dorsal del tabique.

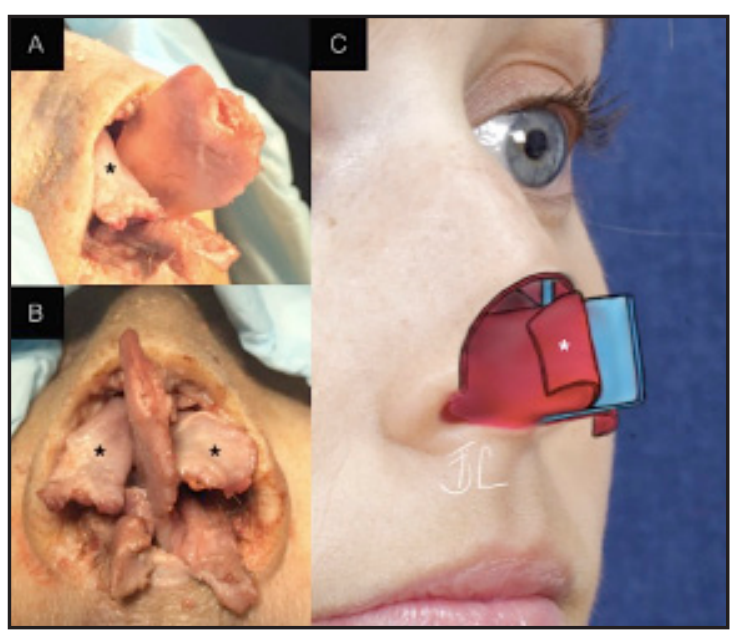

Figura 8. Colgajo compuesto condromucoso septal rotatorio. 8A-B-C: Liberación de un colgajo de mucopericondrio bilateral $\left({ }^{\star}\right)$ que se refleja lateralmente para dar el revestimiento interno al domo nasal. es diseñado con la mayor amplitud posible conservando un marco dorsal de tabique adecuado, extendiéndolo hacia posterior para cubrir todo el tabique cartilaginoso logrando un colgajo de $5 \mathrm{~cm}$ de longitud y $3 \mathrm{~cm}$ de ancho ${ }^{1}$. Desde el ángulo antero inferior del defecto cercano a la espina nasal se crea un túnel de mucopericondrio, necesario para la resección del punto de rotación del colgajo (Figura 6).

Posteriormente se utiliza un osteótomo de $4 \mathrm{~mm}$ para resecar un segmento de $2 \mathrm{~cm}$ de largo de la porción anterior del tabique en el punto de rotación del colgajo, preservando la espina nasal (Figura 6). Una vez completada esta etapa el colgajo es rotado en $90^{\circ}$ desde su base en dirección antero caudal hasta que el borde inferior del colgajo se ubica contra el remanente del marco dorsal del tabique (Figura 7). Finalmente desde el extremo caudal del colgajo ya posicionado se libera un colgajo de mucopericondrio bilateral que se refleja lateralmente para dar el revestimiento interno al domo nasal (Figura 8). El cartílago sobrante denudado que se extiende por sobre el plano de proyección de la nariz es resecado para futuros injertos de estructura (Figura 9).

\section{Colgajo de mucoperiosteo de cornete inferior y medio}

Para defectos limitados de mucosa nasal se pueden crear colgajos a partir de los cornetes medio e inferior. Esta mucosa constituye una red vascular rica dependiente de la arteria esfenopalatina en su porción posterior y media y de la arteria angular en su porción más anterior, aporte suficiente para lograr un colgajo pediculado anterior dependiente de esta irrigación. Ambos colgajos se obtienen a través de una técnica similar. Se comienza con la medialización del cornete a utilizar exponiendo el meato respectivo. Mediante un osteótomo se procede a la realización de perforaciones en la inserción del cornete a la pared lateral. Posteriormente se realiza una incisión en el borde anterior del cornete a través de la cual el componente óseo del cornete dador es removido dejando un colgajo 


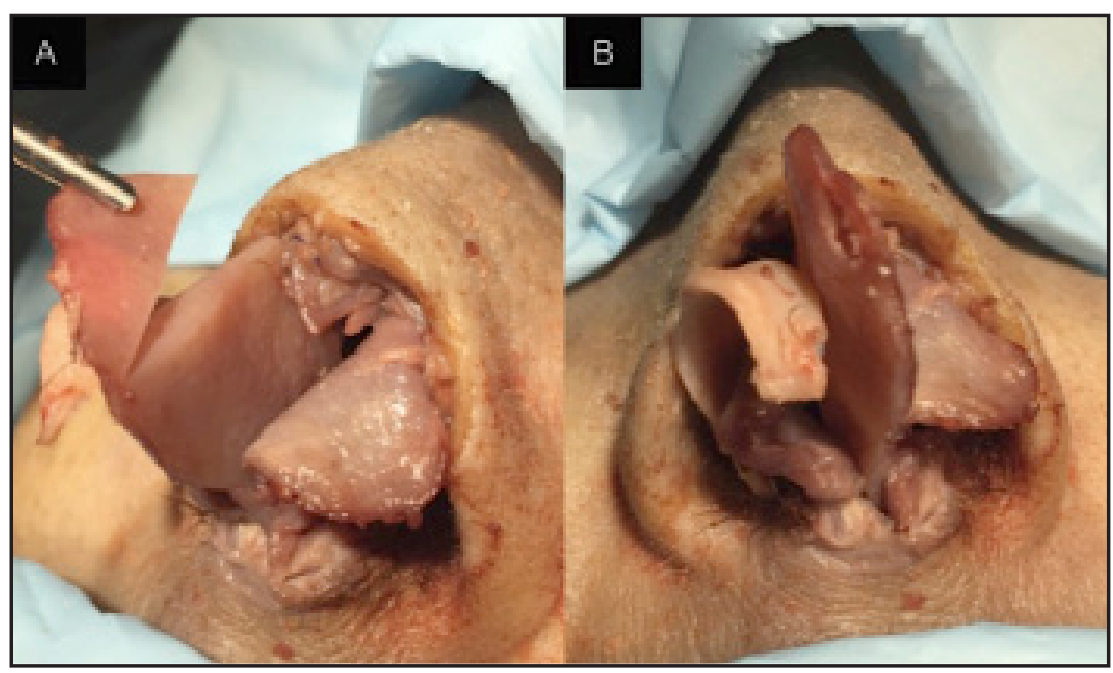

Figura 9. Colgajo compuesto condromucoso septal rotatorio. 9A-B: El cartílago sobrante denudado que se extiende por sobre el plano de proyección de la nariz es resecado para futuros injertos de estructura.

de mucosa, que comprende la cara medial y parte de la cara meatal, que se transfiere y sutura al sitio de defecto (Figura 10).

\section{Colgajo pericráneo frontal}

Este es un colgajo utilizado cuando no se tienen disponibles las estructuras anatómicas anteriormente descritas, en especial cuando han sido resecadas durante una cirugía por invasión tumoral. Este colgajo es ampliamente utilizado en cirugía reconstructiva de base de cráneo anterior ${ }^{7}$.

Este colgajo presenta una excelente vascularización proveniente de las ramas profundas de las arterias supratrocleares y supraorbitarias. Además, presenta características de delgadez y flexibilidad que lo hacen cumplir los requisitos necesarios para el revestimiento interno.

Estudios en cadáveres han demostrado que la principal fuente de vascularización de este colgajo

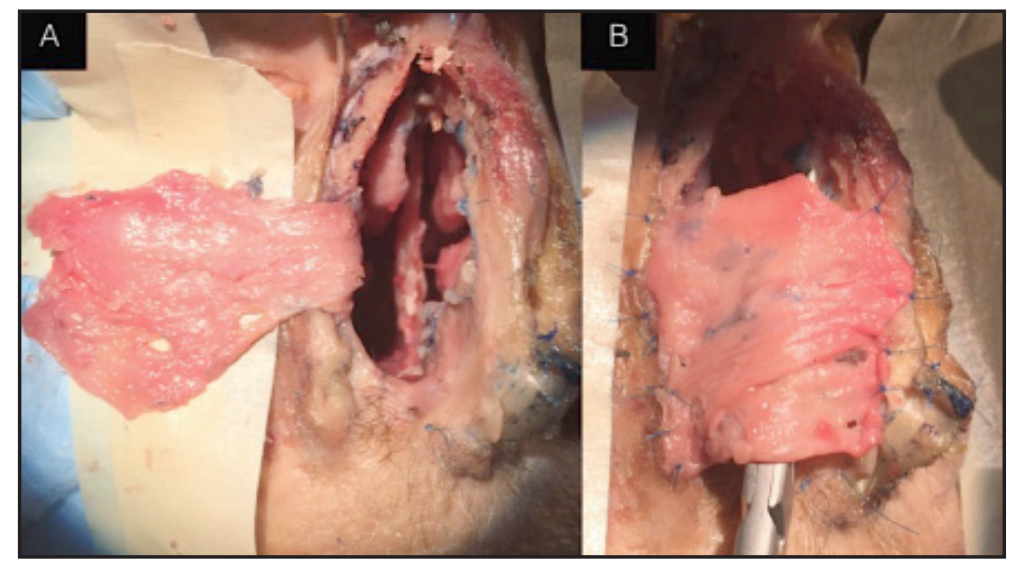

Figura 10. Colgajo de mucoperiosteo de cornete inferior. 10A: Colgajo de mucoperiosteo de cornete inferior reflejado. 10B: Colgajo suturado en ambos bordes del defecto formando el revestimiento interno en el sitio deseado. 
es la arteria supraorbitaria, por lo que debe ser incluida en la base del flap. Las arterias se dividen con una media de $8 \mathrm{~mm}$ y con un máximo de 11 $\mathrm{mm}$ en superficiales y profundas, por lo que la literatura es clara que cuando se diseca esta zona, se debe tener especial precaución en conservar la arteria supratroclear $20 \mathrm{~mm}$ sobre el reborde orbitario.

Este flap no es recomendado en reconstrucciones de más de $70 \mathrm{~mm}$ debido a que la rama profunda supraorbitaria tiene en promedio $70 \mathrm{~mm}$ de longitud en el pericráneo, por lo que un colgajo mayor de esta longitud puede poner en riesgo la vitalidad distal de éste.

Este puede ser realizado vía coronal o vía incisión del colgajo frontal (indiano), debiéndose respetar hasta $15 \mathrm{~mm}$ sobre el reborde orbitario para asegurar la vascularización ${ }^{8}$.

\section{DISCUSIÓN}

La superficie nasal está conformada por subunidades que deben ser tomadas en cuenta al reconstruir una nariz, la forma de estas subunidades está dada por la relación entre los tejidos blandos y su soporte. Muchas veces una tarea más compleja que reconstruir la parte visible, es arreglar de forma adecuada el revestimiento interno nasal. Este debe cumplir con las características necesarias de grosor, maleabilidad y una profusa vascularización. Sobre un revestimiento que cumpla con estas características, podremos reconstruir las siguientes capas asegurándonos un resultado satisfactorio.

Los injertos cartilaginosos utilizados dependen de la vascularización del revestimiento interno y éste necesita del soporte entregado por los injertos. Esta relación denominada como un "romance" por Burget se mantiene hasta hoy ${ }^{6}$.

Las diferentes opciones de revestimiento interno realizadas en nuestros modelos ex vivo han sido ampliamente descritos en la literatura con excelentes resultados. Estos deben ser elegidos cuidadosamente dependiendo del defecto a reparar.

El cirujano otorrinolaringólogo se encuentra en gran ventaja frente a estas técnicas, debido a su competencia en la anatomía intranasal y su instrucción en septoplastía y rinoseptoplastías.

Las diferentes opciones de reconstrucción de revestimiento interno fueron realizadas exitosamente en los especímenes. Desde la perspectiva de Ios autores esta etapa es obligatoria en el desarroIlo de las habilidades quirúrgicas en reconstrucción nasal. Con esto se busca facilitar la realización de estas técnicas sin mayor complejidad posterior.

\section{CONCLUSIÓN}

El revestimiento interno es de suma importancia en la reconstrucción nasal. Todas las técnicas fueron realizadas con éxito en los especímenes. Esta es una primera fase en el desarrollo y aprendizaje de la reconstrucción nasal.

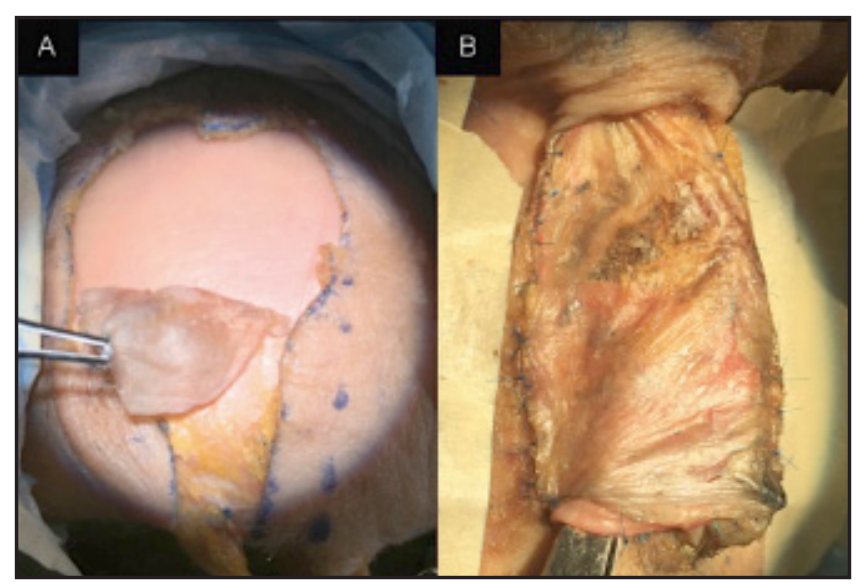

Figura 11. Colgajo pericráneo frontal. 11A: Obtención de colgajo de pericráneo frontal. 11B: Posicionamiento del colgajo en relación al defecto nasal y sutura a remanente de revestimiento interno. 


\section{BIBLIOGRAFÍA}

1. BakeR S. Intranasal lining flaps. Operative Techniques in Otolaryngology 2011 22: 72-83.

2. Giugliano C, Andrades PR, Benitez S. Nasal reconstruction with a forehead flap in children younger than 10 years of age. Plast Reconstr Surg 2004; 114(2): 316-25.

3. Menick $F$. The Evolution of Lining in Nasal Reconstruction. Clin Plastic Surg 2009; 36: 421-41.

4. Kaffenberger BH. The effect of climate change on skin disease in North America. J Am Acad Dermatol 2016; 32: 15-9.

5. Yalamanchili H, Sclafani AP, Schaefer SD, Presti P. The path of nasal reconstruction: from ancient India to the present. Facial Plast Surg 2008; 24(1): 3-10.
6. Burget GC, Menick FJ. Nasal support and lining: the marriage of beauty and blood supply. Plast Reconstr Surg 1989; 84(2): 189-202.

7. Balagopal PG, George NA, Autth R. Anterior skull base reconstruction after cranio-facial resections using galeal pericranial flap. Gulf $J$ Oncolog 2014; 1: 84-8.

8. Sertel S, Pasche P. Pericranial Flap for Inner Lining in Nasal Reconstruction. Ann Plast Surg 2016; 77(4): 425-32.

9. Aneeshimumar MK, Chueng K, Hart R, y cols. Pivoted composite nasal septal flap for reconstruction of the nose. Eur Arch Otorhinolaryngol 2013; 270(9): 2445-50.

10. QueTz J. Update on the septal pivot flap. Facial Plast Surg 2014; 30(3): 300-5. 